\title{
TRATAMENTO DA TUBERCULOSE DA COLUNA VERTEBRAL: CONSERVADOR OU CIRÚRGICO?
}

\author{
TREATMENT OF SPINAL TUBERCULOSIS: CONSERVATIVE OR SURGICAL?
}

\author{
Rodrigo Serikawa de Medeiros ${ }^{1}$, Rodrigo Calil Teles Abdo ${ }^{1}$, Fabiano Cortesi de Paula ${ }^{1}$, Douglas Kenul Narazaki ${ }^{2}$, \\ Leonardo dos Santos Correia², Marcelo Poderoso de Araúuo ${ }^{3}$, Alexandre Fogaça Cristante ${ }^{4}$. Alexandre Sadao lutaka4, \\ Raphael Martus Marcon ${ }^{5}$, Reginaldo Perillo Oliveira 6 , Tarcísio Eloy Pessoa de Barros Fillio
}

\begin{abstract}
RESUMO
Desde a primeira descrição por Percivall Pott da tuberculose de coluna vertebral, tem-se evoluído muito na abordagem dessa doença. Porém ainda existem muitas controvérsias em relação à prevenção das deformidades. Os objetivos de nosso estudo são avaliar as características clínicas e radiológicas de pacientes com tuberculose de coluna e comparar o tratamento conservador isolado com o cirúrgico associado a antibioticoterapia, principalmente quanto à deformidade residual e déficit neurológico. Nosso trabalho é retrospectivo com avaliação de prontuários e radiografias iniciais e ao final do seguimento. O quadro neurológico foi avaliado através da escala da ASIA e as deformidades foram medidas pelo método de Cobb. Foram avaliados 38 pacientes, 11 pacientes foram operados e 27 receberam somente antibioticoterapia. 15 pacientes tinham déficit neurológico, todos melhoraram independente do tipo de tratamento. A média de cifose torácica focal e regional no início do seguimento foi respectivamente $48,8^{\circ}$ e $47,86^{\circ}$. Houve aumento da deformidade na região torácica, segmento mais acometido, de $6,3^{\circ}$ focal e 9,8 regional ao final de 5 anos. O tipo de tratamento não influenciou na progressão da cifose. Paciente com menos de 15 anos e cifose maior que $30^{\circ}$ tiveram pior prognóstico quanto à progressão da deformidade.
\end{abstract}

Descritores: Tuberculose; Coluna vertebral; Cifose.

\section{SUMMARY}

Much has evolved since Percivall Pott's first description of vertebral tuberculosis. However, there still is much controversy regarding the best approaches to prevent deformities. The objectives of this study were to evaluate the clinical and X-ray characteristics of patients with vertebral tuberculosis and to compare the conservative treatment alone to the surgical one associated with antibiotic therapy, particularly regarding residual deformity and neurological deficit. A retrospective evaluation of baseline and end-point X-ray studies and of the medical files was performed. The neurological status was evaluated by using the ASIA scale and the deformities were measured using the Cobb method. Thirty-eight patients were evaluated: 11 were surgically treated and 27 received only antibiotics. Fifteen patients presenting neurological deficit showed improvement regardless of the treatment method employed. The mean focal and regional thoracic kyphosis at baseline was $48.8^{\circ}$ and $47.86^{\circ}$, respectively. An increased incidence of thoracic deformity was found, also being the most affected segment, from $6.3^{\circ}$ focal and $9.8^{\circ}$ regional after 5 years. The type of treatment has not interfered on kyphosis progression. Patients below the age of 15 and with kyphosis above $30^{\circ}$ had worse prognosis regarding deformity progression.

Keywords: Tuberculosis; Spine; Kyphosis.

Citation: Medeiros RS, Abdo RCT, de Paula FC, Narazaki DK, Correia LS, Araújo $M P$ et al. Treatment of spinal tuberculosis: conservative or surgical? Acta Ortop Bras. [serial on the Internet]. 2007; 15(3): 128-131. Available from URL: http://www. scielo.br/aob. MP et al. Tratamento da tuberculose da coluna vertebral: conservador ou cirúrgico? Acta Ortop Bras. [periódico na Internet]. 2007; 15(3):128-131. Disponível em URL: http://www.scielo.br/aob.

\section{INTRODUÇÃO}

Em 1779, Percivall Pott(1), em sua monografia, foi o primeiro a fazer a associação entre a tuberculose e a doença da coluna vertebral. Descreveu o diagnóstico nosológico e sindrômico. Observou que pacientes com paralisia e espasticidade dos membros inferiores tinham como causa uma curvatura anormal da coluna. O início do quadro era insidioso, sendo mais rápido no adulto. $O$ estado geral pouco se alterava. A incisão posterior paramediana no dorso e a drenagem de material espesso contido nas vértebras determinava a melhora funcional dos membros acometidos, permitindo os pacientes deambular depois de 6 semanas. Notou ainda que as vértebras estavam cariadas, com seu corpo aumentado e mais esponjoso.
A tuberculose de coluna é a tuberculose óssea mais freqüente, correspondendo à 50\%. Pode estar associada à doença pulmonar ou não. Se não diagnosticada e tratada precocemente pode levar a seqüelas importantes como paraplegia e deformidades, que determinam perda funcional.

Quanto ao tratamento, a literatura ainda é controversa. Compere e Jerome, Chandler e Page, e Cleveland apud Baley et al. ${ }^{(2)}$ descreveram sua experiência da artrodese de coluna via posterior. Bennett, Fallen e Kaplana apud Baley et al. (2) apresentaram seus resultados com tratamento conservador. Todos com bom resultados, porém nenhum comparativo. Kidner e Muro, Mayer e Adams apud Baley et al. ${ }^{(2)}$ compararam o tratamento conservador com a artrodese posterior em crianças, concluindo que nenhum tratamento foi superior ao outro na fase aguda da doença. No entanto, no estágio

Trabalho realizado no Instituto de Ortopedia e Traumatologia do Hospital das Clínicas da Faculdade de Medicina da Universidade de São Paulo

Endereço para correspondência: Douglas Kenji Narazaki - Rua Carneiro da Cunha, 675, ap 115, CEP 04144-001, São Paulo - SP - Brasil - Fone: 82978139 - Email: dogkn@ig.com.br

1. Residente em Ortopedia e Traumatologia do HC/FMUSP

2. Estagiário do Grupo de Coluna do Instituto de Ortopedia e Traumatologia do HC/ FMUSP

3. Médico Preceptor do Instituto de Ortopedia e Traumatologia do HC/ FMUSP

4. Médico Assistente do Instituto de Ortopedia e Traumatologia do HC/ FMUSP

5. Pós-Graduando no Departamento de Ortopedia e Traumatologia do HC/FMUSP

6. Médico Assistente e Chefe do Grupo de Coluna do Instituto de Ortopedia e Traumatologia do HC/ FMUSP

7. Professor Titular do Departamento de Ortopedia e Traumatologia da Faculdade de Medicina da Universidade de Sáo Paulo

Trabalho recebido em 28/09/06 e aprovado em: 10/10/06 